\title{
Hydrogen Behavior in an Ultrafine-Grained Electrodeposited Pure Iron
}

\author{
Yuhua SU, ${ }^{1)}$ Yo TOMOTA, ${ }^{2)}$ Junichi SUZUKI ${ }^{3)}$ and Masato OHNUMA ${ }^{4)}$ \\ 1) Graduate Student of Ibaraki University, 4-12-1 Nakanarusawa, Hitachi, Ibaraki, $316-8511$ Japan. E-mail: \\ 09nd607l@hcs.ibaraki.ac.jp $\quad$ 2) Graduate School of Science and Engineering, Ibaraki University, 4-12-1 Nakanarusawa, \\ Hitachi, Ibaraki, 316-8511 Japan. E-mail: tomota@mx.ibaraki.ac.jp 3) Research Center for Neutron Science and \\ Technology, Comprehensive Research Organization for Science and Society (CROSS), Tokai, Ibaraki, 319-1106 Japan. E-mail: \\ j suzuki@cross.or.jp 4) National Institute for Materials Science, 1-2-1 Sengen, Tsukuba, 305-0047 Japan. E-mail: \\ OHNUMA.Masato@nims.go.jp
}

(Received on April 4, 2011; accepted on May 20, 2011)

\begin{abstract}
The behavior of hydrogen in an ultrafine-grained electrodeposited pure iron with Lankford ( $r$ ) value larger than 7.0 was studied by small angle neutron scattering (SANS) and thermal desorption spectroscopy (TDS). Nano-sized inhomogeneity consisting of hydrogen bubble exists in the deposited specimen. The bubble size increases a little by $673 \mathrm{~K}$ annealing and then all the bubbles disappear after $973 \mathrm{~K}$ annealing. With rolling at room temperature (RT), the bubble size and number density are found to decrease, which must be caused by the change in the status of hydrogen location during plastic deformation. Crystal rotation and grain coalescence are revealed to occur after rolling deformation from electron backscattered diffraction (EBSD) results.
\end{abstract}

KEY WORDS: electrodeposited pure iron; small angle neutron scattering; hydrogen; annealing; cold rolling.

\section{Introduction}

Hydrogen is easily introduced into metals and alloys during electrodeposition process and normally has negative influence on the quality of the deposits. ${ }^{1,2}$ It is not easy to experimentally detect hydrogen because of its low sensitivity to external stimulations, high mobility and small solubility. Therefore, the understanding of hydrogen behavior including diffusion and trapping has been limited due to the lack of suitable monitoring tools. ${ }^{3)}$ Thermal desorption spectroscopy (TDS) is usually used to determine the hydrogen desorption characteristics, i.e., trapped sites and total amounts of hydrogen over the entire specimen. ${ }^{4-6)}$ Information about the size, shape, number and distribution of hydrogen cluster or bubble are still uncertain. Small angle neutron scattering (SANS) is an appropriate technique for measuring microstructure in a scale from several nanometers to micrometers for many materials and has advantage of high sensitivity to light elements like hydrogen as neutron interacts with nuclei. ${ }^{7-9)}$ In the present work, SANS and TDS were employed to investigate the hydrogen behavior in an electrodeposited pure iron with annealing or cold rolling. It has been reported that this electrodeposited pure iron sheets show the Lankford (r) value larger than 7.0. ${ }^{10-13)}$ The reason of such unusual anisotropic plastic deformation is also discussed.

\section{Experimental Procedures}

Ultra-fine crystalline pure iron sheet used in this study was produced through industrial process by TohoZinc Co. Ltd., with purity of more than 99.995 wt.\%. As is observed in Fig. 1, microstructure of the as-deposited sheet consists of columnar grains with the cross section diameter of smaller than $1 \mu \mathrm{m}$ (see (a) and (d)). The uni-directionally oriented columnar grains exhibited extremely sharp $<111>/$ ND fiber texture ((Figs. 1(b), 1(c)). TEM microstructure reported in ref.12 ((Fig. 1(d)) suggests the existence of stress field. Some sheets with $1.5 \mathrm{~mm}$ in thickness were annealed at 673 $\mathrm{K}$ or $973 \mathrm{~K}$ for $3.6 \mathrm{ks}$ and other $1.1 \mathrm{~mm}$ thick sheets were cold rolled at RT by $10 \%, 20 \%$ or $40 \%$ reduction in area. Scanning electron microscope (SEM) of Hitachi 4300, equipped with a TSL electron backscatter diffraction (EBSD) system was used for observing the top surface and cross section of the as-deposited specimens before and after deformation, and operated at an accelerating voltage of 15 $\mathrm{kV}$ and a tilt angle of $70^{\circ}$ with step size of $0.1 \mu \mathrm{m}$.

TDS of hydrogen was conducted for the deposited and deformed specimens with a heating rate of $8 \mathrm{~K} / \mathrm{s}$ in a temperature range from $\mathrm{RT}$ to $1073 \mathrm{~K}$ in a vacuum chamber, where $10 \mathrm{~mm} \times 10 \mathrm{~mm} \times 1 \mathrm{~mm}$ specimens were prepared. ${ }^{5)}$

Specimen size for SANS measurement was $20 \mathrm{~mm} \times$ $20 \mathrm{~mm} \times 2 \mathrm{~mm}$. SANS measurements were carried out with a small-angle neutron scattering spectrometer SANS-J-II equipped at the cold neutron source of JRR-3, JAEA. A magnetic field of 1T was applied to separate nuclear scattering component from magnetic scattering component. And the scattered intensity was recorded by a two-dimensional detector which could be positioned at distances of $2.5 \mathrm{~m}$ and $10 \mathrm{~m}$ from the specimen. The incident wave length was set 

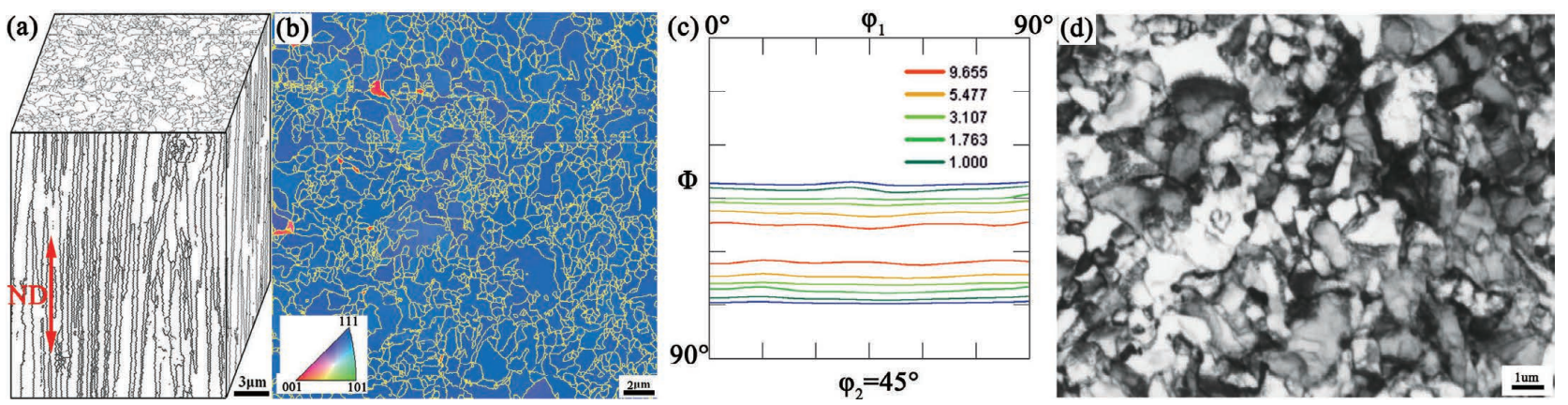

Fig. 1. Microstructures of the as-deposited pure iron: (a) grain boundary characteristics determined by EBSD, (b) inverse pole figure map on the top surface, where the grain boundary with corlor in yellow includes misorientation angle higher than $2^{\circ}$, (c) ODF $\left(\varphi_{2}=45^{\circ}\right.$, cross section) obtained by EBSD and (d) TEM micrograph ${ }^{(10)}$.

to be $0.656 \mathrm{~nm}$, which provided the scattering vector $\mathrm{q}(\mathrm{q}=$ $4 \pi \sin \theta / \lambda, 2 \theta$ is the scattering angle, with neutron wave length of $\lambda$ ) range of $5 \times 10^{-3} \mathrm{~nm}^{-1} \leq \mathrm{q} \leq 1.99 \mathrm{~nm}^{-1}$, corresponding to real structure sizes of the order of 3-1 $000 \mathrm{~nm}^{7,14)}$ The calibration to absolute intensity was performed by measurement of the irradiated aluminum standard specimen.

\section{Results and Discussion}

\subsection{Results of TDS}

Figure 2 shows hydrogen desorption profiles obtained from the TDS for the as-deposited and the $40 \%$ rolled specimen. There are three main peaks (1), (2) and (3) on the asdeposited specimen spectra at about $473 \mathrm{~K}, 723 \mathrm{~K}$ and $953 \mathrm{~K}$, respectively. All peaks are found to shift left and the intensity around peak (2) becomes stronger while that around peak (3) weaken after $40 \%$ cold rolling. In addition, the peak (2) and (3) seems to show split. It indicates that hydrogen distribution in the specimen was changed by cold rolling. It is reported that the activation energies for hydrogen evolution from different trapping sites in pure iron increases in the order of grain boundaries, dislocations and microvoid, and that hydrogen is claimed to exist in the microvoids as a molecule. ${ }^{15)}$ After cold rolling, the lowest hydrogen release peak (1) shifted toward left, and intensity of peak (2) on TDS profile increased in Fig. 2, which may correspond to hydrogen release from dislocation. On the other hand, the peak (3) related to hydrogen release from microvoid becomes much smaller. As the relative intensity of peak (2) becomes higher while that of peak (3) decreases in the $40 \%$ rolled specimen. It suggests that hydrogen trap site changed from microvoid to dislocation. The reasons for peak split at peak (2) and (3) must stem from some transition stages but are not clear at the moment.

\subsection{Effect of Annealing on SANS Profile}

The SANS intensity in a magnetic field is composed of an isotropic nuclear contribution and an anisotropic magnetic contribution. The nuclear and magnetic components can be separated from each other using the following formula, ${ }^{16-19)}$

$$
I(q)=I_{\text {Nuclear }}+\sin ^{2} \alpha \cdot I_{\text {Magnetic }}(q)
$$

Where, $\alpha$ is the angle between the scattering vector $q$ and the magnetic field direction. The nuclear component of the scattering intensity can be determined at $\alpha=0^{\circ}$, while the sum of nuclear and magnetic intensity is found at $\alpha=90^{\circ}$.

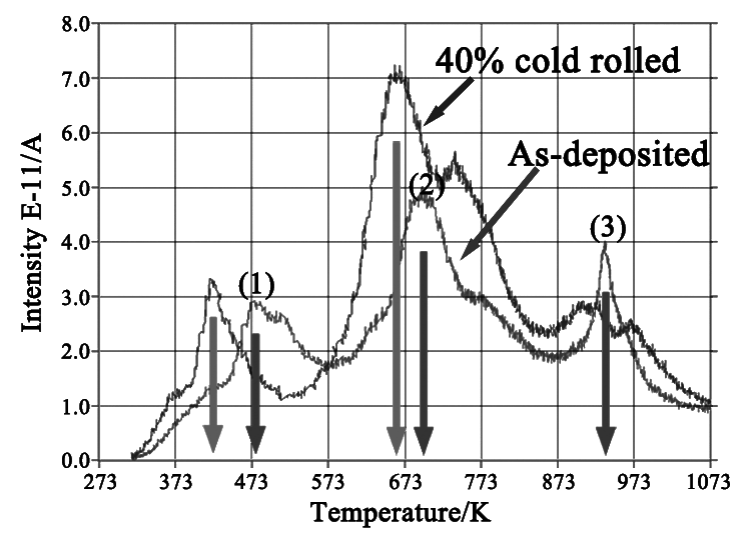

Fig. 2. Hydrogen TDS spectra obtained from the as-deposited and cold rolled specimens.

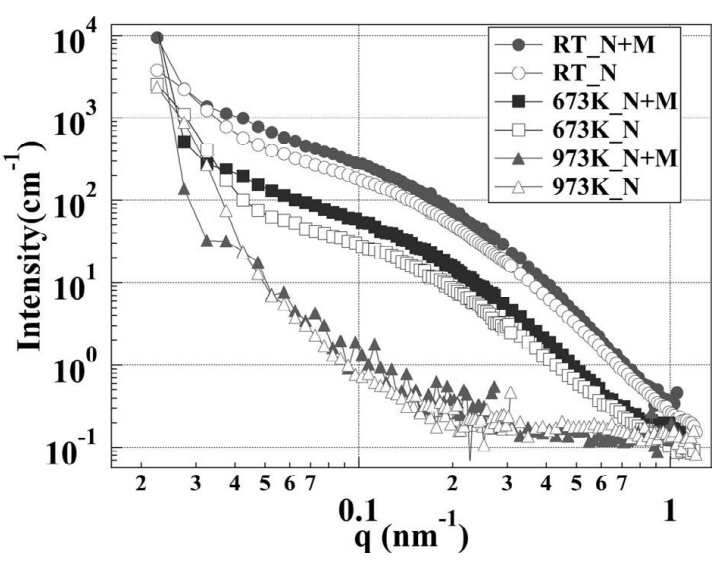

Fig. 3. Effect of annealing on SANS profiles: $N$ and $M$ refer to nuclear scattering and magnetic scattering, respectively.

Figure 3 shows SANS profiles for the annealed specimens with a magnetic field of 1T. As seen, scattering intensity decreases with increasing of annealing temperature. So called "Guinier region" caused by the existence of nano-size imhomogeneity which causes SANS scattering contrast, for example, hydrogen bubble, is evidently observed in nuclear scattering profiles for the as-deposited and the $673 \mathrm{~K}$ annealed specimens, while not for the $973 \mathrm{~K}$ specimen. This indicates the disappearance of inhomogeneity by annealing at $973 \mathrm{~K}$. It is interesting to compare this result with r-values reported in ref.12 (7.6 for the as-deposited, 5.2 for the $673 \mathrm{~K}$ annealed and 2.2 for the $973 \mathrm{~K}$ annealed specimen). The average particle radius determined from the fitting with 
Guinier approximation $^{20,21)}$ were about $15 \mathrm{~nm}$ commonly for the as-deposited and $673 \mathrm{~K}$ annealed specimens. A remarkable change in SANS profile for the $973 \mathrm{~K}$ annealed specimen may be ascribed to a few reasons, i.e., defects like void, impurity elements like $\mathrm{C}$ and $\mathrm{N}$, and hydrogen. By annealing at $973 \mathrm{~K}$, the microstructure changes from fine columnar grains to coarse equiaxed grains as was reported in ref.12. This change in grain size and shape is postulated to affect the SANS intensity at a lower $q$ region than that in the measured region. If carbides or nitrides precipitate by annealing, this would increase the SANS intensity. Hence, the drastic decrease in the SANS intensity observed at $q=0.02-1 \mathrm{~nm}^{-1}$ in Fig. 3 is believed to be caused mostly by the hydrogen desorption from comparison between Figs. 2 and 3.

The following equation can be used for profile fitting to get the particle size distribution ${ }^{19-21)}$ assuming spherical inhomogeneity (independent spherical particles model).

$$
I(q)=\Delta \rho^{2} N_{d} \int_{0}^{\infty} V^{2}(R) F^{2}(q, R) N(R) d R
$$

In which, $\Delta \rho$ is the scattering length contrast between the particle and the matrix, $F(q, R)$ is particle shape factor, $N_{d}$ is number density of the particle, $V(R)$ is the volume of the particle. For spherical particle, the shape factor $F(q, R)$ can be written as,

$$
F(q, R)=\left(\frac{3[\sin (q R)-q R \cos (q R)]}{(q R)^{3}}\right)
$$

Eq. 4 depicts the log-normal normalised size distribution function,

$$
N(R)=\frac{1}{R \sigma \sqrt{2 \pi}} \exp \left(-\frac{\left(\ln R-\ln R_{1}\right)^{2}}{2 \sigma^{2}}\right)
$$

Where $R$ is the particle radius, $R_{l}$ is the median radius and $\sigma$ is the variance of the distribution. After fitting, particle information including average size $\left(R_{\text {Ave }}\right)$, size distribution $(N(R))$, number density $\left(N_{d}\right)$, the volume of the particle and volume fraction $\left(V_{f}\right)$ can be calculated by the following equations.

$$
\begin{aligned}
& R_{\text {Ave }}=\frac{\int_{0}^{\infty} N(R) R d R}{\int_{0}^{\infty} N(R) d R} \\
& V_{R}=\frac{\int_{0}^{\infty} \frac{4}{3} \pi R^{3} N(R) d R}{\int_{0}^{\infty} N(R) d R} \\
& V_{f}=N_{d} * V_{R}
\end{aligned}
$$

The fitted profiles for the deposited and the $673 \mathrm{~K}$ annealed specimens were depicted in Fig. 4 showing good coincidence with the measurements, in which bubble quantity was computed based on the difference of scattering length density contrast $(\Delta \rho)$ between hydrogen bubble assuming $1 \mathrm{~atm}\left(0^{\circ} \mathrm{C}, 101.325 \mathrm{kPa}\right)$ and the iron matrix. Scattering length density is defined as:

$$
\rho=\frac{\sum_{i=1}^{n} b_{c i}}{V_{m}}
$$
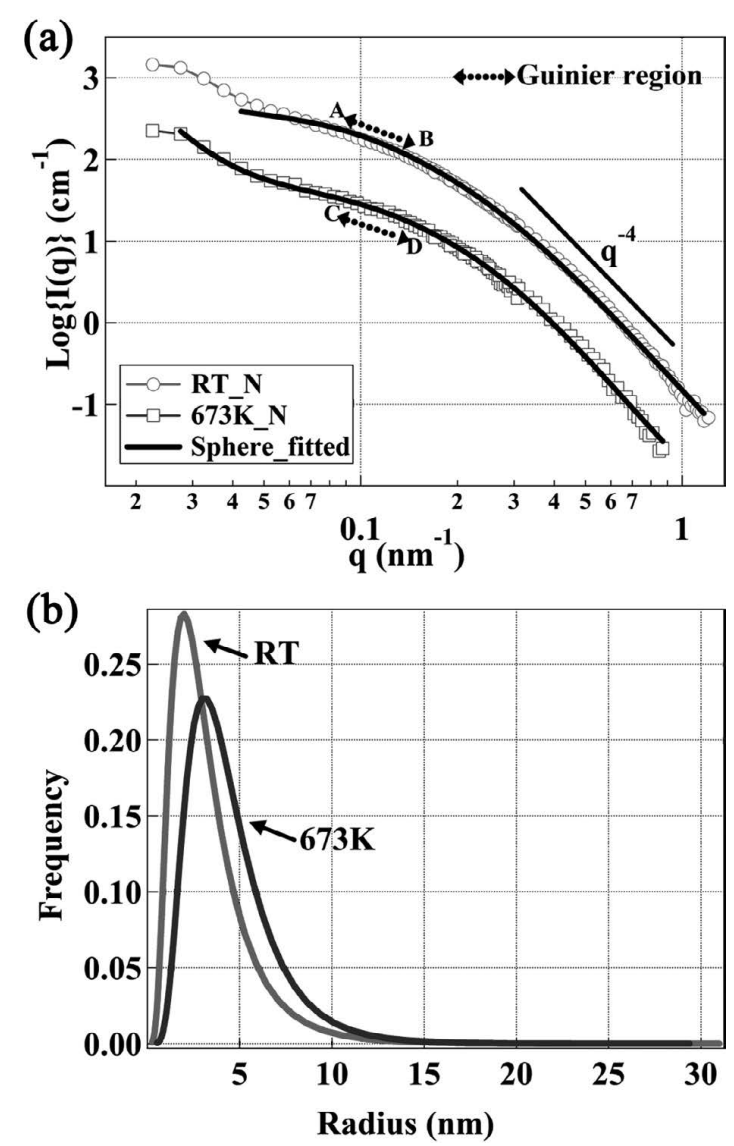

Fig. 4. Results of SANS profile analysis: (a) sphere particle model fitting and (b) log-normal size distribution obtained from the curve fitting.

$$
\frac{1}{V_{m}}=N_{A} * \frac{\rho_{0}}{A}
$$

Where $b_{c i}$ is the bound coherent scattering length of ith of $\mathrm{n}$ atoms in a molecular with molecular volume $V_{m}, N_{A}$ is Avogadro constant, $\rho_{0}$ is density of molecule and $A$ means relative atomic or molecule mass.

For hydrogen molecule, $\rho_{0}=0.08988 \mathrm{~g} / \mathrm{L}, A=2.016$ $\mathrm{g} \cdot \mathrm{mol}^{-1}, b=\left(-3.739 \times 10^{-13} \mathrm{~cm}\right) \times 2$, while for iron, $\rho_{0}=$ $7.874 \mathrm{~g} \cdot \mathrm{cm}^{-3}, A=55.845 \mathrm{~g} \cdot \mathrm{mol}^{-1}, b=9.45 \times 10^{-13} \mathrm{~cm}$, the calculated scattering length density is $\rho_{H_{2}}=-2.02 \times 10^{7} \mathrm{~cm}^{-2}$ and $\rho_{\mathrm{Fe}}=8.02 \times 10^{10} \mathrm{~cm}^{-2}$, respectively.

$$
\Delta \rho=\rho_{\mathrm{H}_{2}}-\rho_{\mathrm{Fe}}
$$

As is shown in Table 1, from SANS results, hydrogen bubble size became larger after $673 \mathrm{~K}$ annealing. However, bubble number density and volume fraction decreased because of hydrogen desorption. The hydrogen volume fraction in the as-deposited specimen estimated by SANS was in excellent agreement with that measured by TDS.

\subsection{Effect of Cold Rolling Deformation on SANS Pro- file}

1 Tesla magnetic field was applied in the horizontal direction during SANS measurement on the cold rolled specimens. As seen in Fig. 5, the nuclear scattering intensity decreases obviously with increase of rolling reduction, and "Guinier region" is observed in all profiles. Fig. 5(a) shows the measured and fitted nuclear scattering profiles for the 
Table 1. Influences of annealing or cold rolling on size, number density and volume fraction of inhomogeniety (hydrogen) measured by SANS and TDS.

\begin{tabular}{|c|c|c|c|c|c|c|c|}
\hline & \multirow{2}{*}{ Specimen } & \multicolumn{4}{|c|}{ SANS by a sphere fitted method } & \multicolumn{2}{|c|}{ TDS } \\
\hline & & Rave/nm & $\mathrm{Vr} / \mathbf{n m}^{-3}$ & $\mathrm{Nd} / \mathrm{cm}^{-3}$ & $\mathbf{V f} / \%$ & Wt./ppm & Vf/\% \\
\hline \multirow[t]{2}{*}{ Annealed } & Deposited & 3.36654 & 470.694 & $1.74906 \mathrm{e}+16$ & 0.823 & 0.09 & 0.788 \\
\hline & $673 \mathrm{~K}$ annealed & 4.49462 & 806.504 & $1.49534 \mathrm{e}+15$ & 0.121 & - & - \\
\hline \multirow[t]{4}{*}{ Cold Rolled } & Deposited & 4.95829 & 1024.59 & $1.77688 \mathrm{e}+16$ & 1.82 & 0.09 & 0.788 \\
\hline & $10 \%$ rolled & 3.53605 & 540.134 & $1.50534 \mathrm{e}+16$ & 0.813 & - & - \\
\hline & $20 \%$ rolled & 2.76265 & 382.475 & $9.23594 \mathrm{e}+15$ & 0.353 & - & - \\
\hline & $40 \%$ rolled & 2.67044 & 357.75 & $2.48938 \mathrm{e}+15$ & 0.089 & 0.11 & 0.963 \\
\hline
\end{tabular}
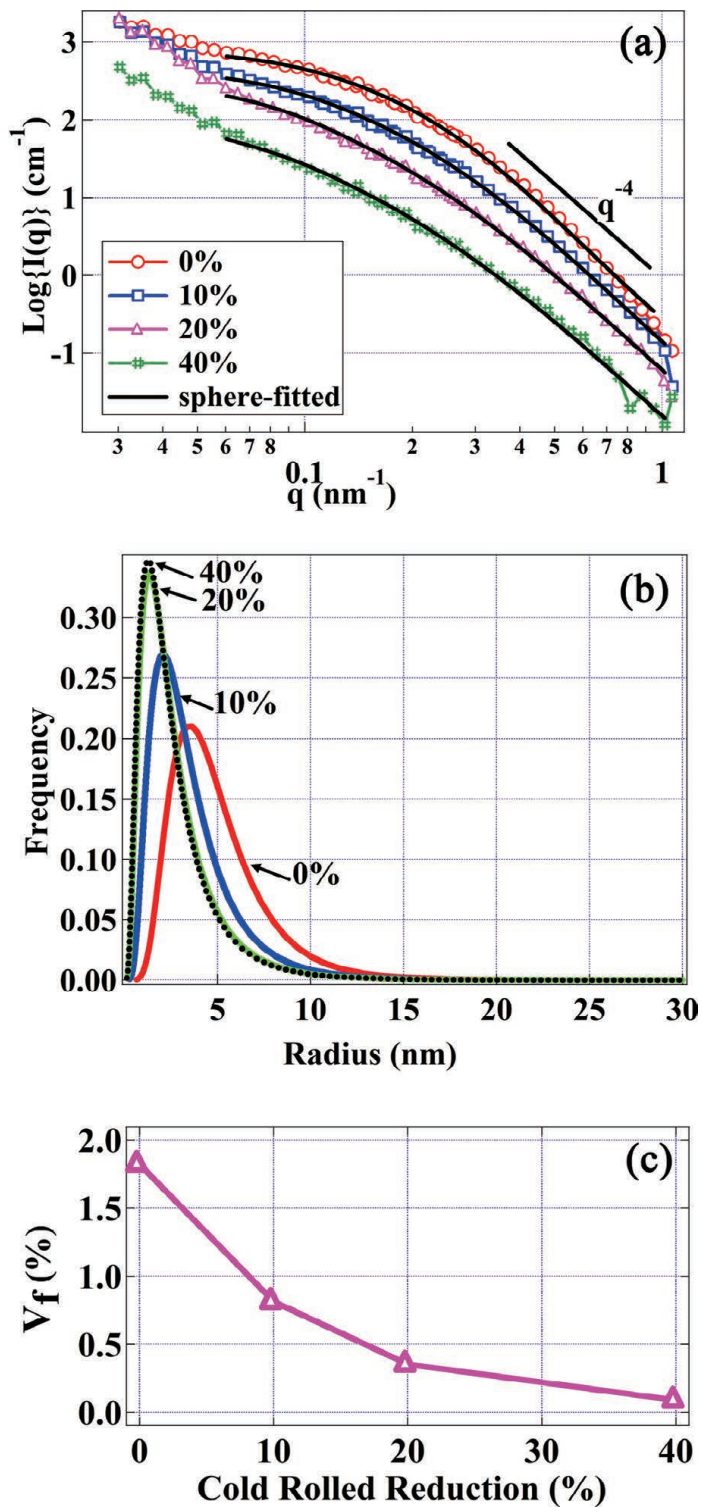

Fig. 5. Effect of cold rolling on inhomogeneity particle: (a) nuclear SANS profiles, (b) size distribution and (c) volume fraction of inhomogeneity.

cold rolled specimens with different rolling reduction. Similarly to the case of annealing described above, corresponding hydrogen bubble information was calculated and the results were presented in Figs. 5(b) and 5(c). It is found that average size, number density and volume fraction are decreased with increase of rolling reduction. Table $1 \mathrm{com}-$ pared hydrogen content of the as-deposited and the $40 \%$

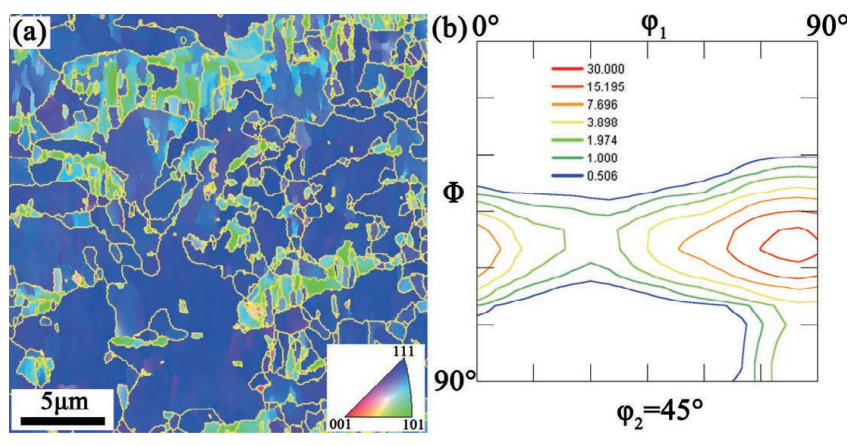

Fig. 6. Microstructure of $40 \%$ rolled specimen observed by EBSD: (a) top surface inverse pole figure map of the as-deposited specimen and (b) ODF of the as-deposited specimen.

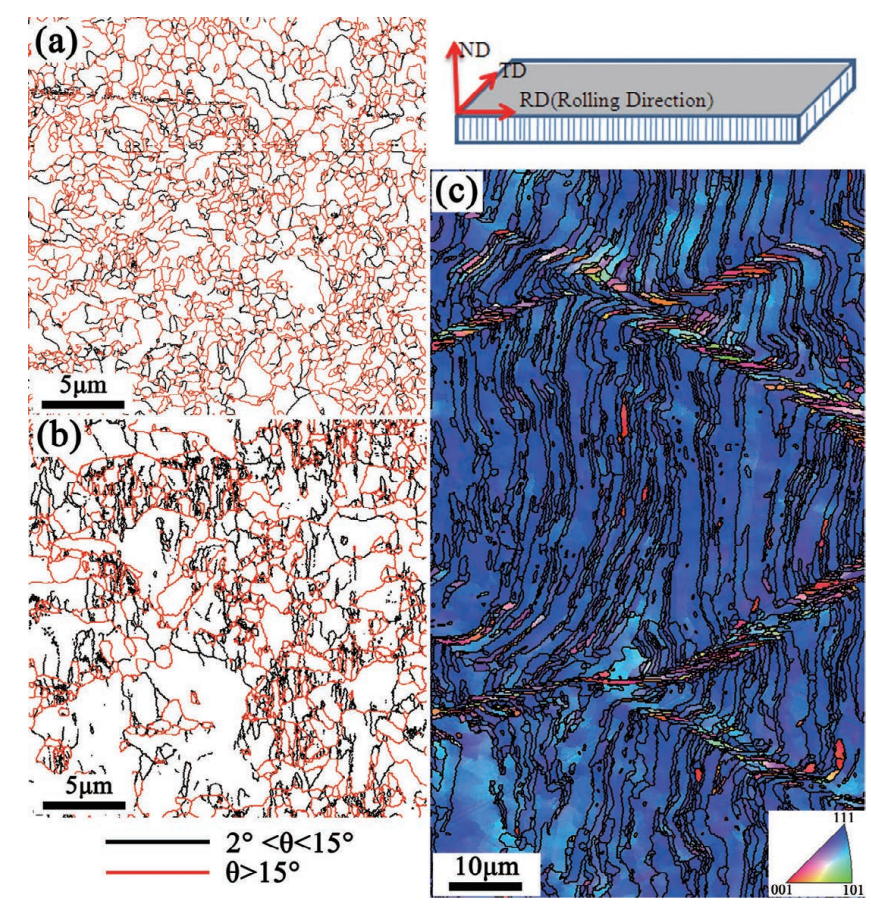

Fig. 7. Change in microstructure with rolling: (a) grain boundary (low-angle grain boundaries with misorientation $2^{\circ}<\theta<15^{\circ}$ and high-angle boundaries above $15^{\circ}$ ) maps on the top surface of the as-deposited specimen by EBSD, (b) that of $40 \%$ rolled specimen and (c) IPF map(ND) in the cross section of the $40 \%$ cold rolled specimen.

rolled specimens determined from SANS and TDS results. For the as-deposited specimen, the SANS result was consistent with that of TDS. However, the amounts of hydrogen in the $40 \%$ rolled specimen determined by SANS data was lower than that from TDS. This is because only hydrogen bubbles were considered in SANS, while all kinds of hydrogen forms including bubble, segregation and so on can be detected and transformed into hydrogen content by TDS. From the amount difference and TDS hydrogen desorption peaks changes observed in Fig. 2, some hydrogen bubbles are likely to transform into other existing forms by cold rolling.

\subsection{Microstructure Change with Cold Rolling}

Figure 6 compares the inverse pole figure map on the surface and corresponding ODF texture calculated from EBSD data. After $40 \%$ rolling, texture component in ND direction hardly changes, while that along the rolling direction the $<112>$ and $<110>$ orientations texture becomes 
stronger. That means strong $\{111\}<\mathrm{hkl}>$ random texture evolved into $\{111\}<112>$ and $\{111\}<110>$ orientation dominant due to lattice rotation. All boundaries are defined as either low-angle grain boundaries with misorientation angle $\theta$ between $2^{\circ}$ and $15^{\circ}$ or high-angle grain boundaries above $15^{\circ}$, grain coalescence is verified from the EBSD grain boundary map on the surface by OIM software shown in Figs. 7(a) and 7(b). ${ }^{22-24)}$ It may come from grain boundary motion by a kind of hydrogen related stress-driven mechanism during deformation. ${ }^{25-28)}$ And the fraction of low angle boundaries increased from about $28 \%$ in the asdeposited to $50.7 \%$ in the $40 \%$ rolled specimen. Deformation bands are observed from the inverse pole figure map of the cross section in Fig. 7(c), revealing that dislocation motion is the main mechanism of plastic deformation of the as-deposited specimen. Grain coalescence of the columnar grain is recognized by comparing Figs. 1(a) and 7(c). As will be reported elsewhere, crystal rotation and grain coalescence are also observed in case of tensile deformation. ${ }^{29)}$ Hence, grain coalescence related with hydrogen behavior during plastic deformation is speculated to realize unusual high r-value.

\section{Conclusions}

Effect of annealing and cold rolling on hydrogen behavior in an electrodeposited pure iron sheet was studied by means of TDS and SANS. The obtained results would be summarized as follows.

(1) Three hydrogen desorption peaks appear on the TDS profile during annealing, at about $473 \mathrm{~K}, 723 \mathrm{~K}$ and $973 \mathrm{~K}$ for the as-deposited specimen. All peaks shift left and two peaks at higher temperatures merge into unity after $40 \%$ cold rolling, which should be caused by the change in the status of hydrogen during the plastic deformation.

(2) From SANS results, nano-sized inhomogeneity is found to exist in the as-deposited and $673 \mathrm{~K}$ annealing specimen. Its size increases a little by $673 \mathrm{~K}$ annealing and then disappear after $973 \mathrm{~K}$ annealing. By using an independent spherical particles model for the SANS data obtained, the hydrogen volume fraction in the as-deposited specimen was estimated was in excellent agreement with that measured by TDS.

(3) With rolling at RT, the bubble size and volume fraction are found to decrease. Compared with TDS results, some hydrogen bubbles are likely to transform into other existing forms by cold rolling.

(4) Strong random texture $\{111\}<\mathrm{hkl}>$ grows into $\{111\}<112>$ and $\{111\}<110>$ orientation dominant after cold $40 \%$ rolling. Meanwhile, grain coalescence occurs dur- ing plastic deformation, which could be related to the extremely high $r$ value.

\section{Acknowledgements}

This study was financially supported by the Grant-in-Aid for Scientific Research on Innovative Area, "Bulk Nanostructured Metals" through MEXT, Japan (contract No.22102006) and the support is gratefully appreciated.

\section{REFERENCES}

1) P. C. Hsu, S. K. Seol, T. N. Lo, C. J. Liu and C. L.Wang: The Electro. Soc., 155 (2008), 400.

2) K. S. Kumar, S. Suresh, M. F. Chisholm, J. A. Horton and P. Wang: Acta Mater., 51 (2003), 387.

3) K. Takai, H. Shoda, H.Suzuki and M. Nagumo: Acta Mater., 56 (2008), 5158.

4) T. Ohmisawa, S. Uchiyama and M. Nagumo: Alloys Compd., 356357 (2003), 290.

5) E. Tal-Gutelmacher, D. Eliezer and E. Abramov: Mater. Sci. Eng., A445-446 (2007), 625.

6) K. Ebihara, T. Suzudo, H. Kaburaki, K. Takai and S. Takebayashi: ISIJ Int., 47 (2007), 1131.

7) G. Muller, M. Uhlemann, A. Ulbricht and J. Bohmert: Nucl. Mater., 309 (2006), 114.

8) M. Ohnuma, J. Suzuki, F. G. Wei and K. Tsuzaki: Scr. Mater., 58 (2008), 142.

9) C. E. Buckley, H. K. Birnbaum, D. Bellmann and P. Staron: Alloys Compd., 293-295 (1999), 231.

10) N. Yoshinaga, D. Vanderschueren, L. Kestens, K. Ushioda and J. Dilewjns: ISIJ Int., 38 (1998), 610.

11) N. Yoshinaga, S. Hiwatashi, K. Ushioda and O. Akisue: CAMP-ISIJ, 12 (1999), 1277.

12) T. Motegi, M. Masuyama, K. Iida, T. Suzuki, H. Sato and Y. Tomota: Tetsu-to-Hagané, 88 (2002), 801.

13) N. Yoshinaga, N. Sugiura and S. Hiwatashi: ISIJ Int., 48 (2008), 667.

14) M. Bischof, P. Staron, A. Michels, P. Granitzer, K. Rumpf and H. Leitner: Acta Mater., 55 (2007), 2637.

15) W. Y. Choo and J. Y. Lee: Metal. Trans. A, 13A (1982), 135.

16) M. Maxelon, A. Pundt, W. Pyckhout-Hintzen, J. Barker and R. Kirchheim: Acta Mater., 49 (2001), 2625.

17) M. Ohnuma, J. Suzuki, S. Ohtsuka, S. W. Kim, T. Kaito, M. Inoue and H. Kitazawa: Acta Mater., 57 (2009), 5571.

18) M. Ojima, M. Ohnuma, J. Suzuki, S. Ueta, S. Narita, T. Shimizu and Y. Tomota: Scr. Mater., 59 (2008), 313.

19) R. Przeniosło, R. Winter, H. Natter, M. Schmelzer, R. Hempelmann and W. Wagner: Physical Review B, 63 (2001), 054408.

20) L. A. Feigin and D. I. Svergun: Structure Analysis by Small-Angle X-ray and Neutron Scattering, Plenum Press, New York, (1987).

21) A. Michels and J.org Weissmuller: Rep. Prog. Phys., 71 (2008), 066501.

22) G. J. Fan, L. F. Fu, H. Choo, P. K. Liaw and N. D. Browning: Acta Mater., 54 (2006), 4781.

23) L. Li, T. Ungar, Y. D. Wang, J. R. Morris, G. Tichy, J. Lendvai, Y. L. Yang, Y. Ren, H. Choo and P. K. Liaw: Acta Mater., 57 (2009), 4988.

24) Y. B. Wang, B. Q. Li, M. L. Sui and S. X. Mao: Appl. Phys. Lett., 92 (2008), 011903.

25) N. Lu, X. Wang, Z. G. Suo and J. Vlassak: Mater. Res., 24 (2009) 379.

26) D. S. Gianola, B. G. Mendis, X. M. Cheng and K. J. Hemker: Mater. Sci. Eng., A483-484 (2008), 637.

27) K. Zhang and J. R. Weertman: Appl. Phys. Lett., 87 (2005), 061921.

$28)$ D. S. Gianola, V. Petegem, M. Legros, S. Brandstetter, H. V. Swygenhoven and K. J. Hemker: Acta Mater., 54 (2006), 2253.

29) Y. H. Su, Y. Tomota, J. Suzuki, S. Harjo and Y. Adachi: unpublished work (to be submitted), (2011). 